\title{
ANZTLA 2013 Statistics
}

by Kerrie Stevens

ANZTLA Statistician

NZTLA Statistics are compiled annually from data provided by ANZTLA member
libraries on a voluntary basis.

The reporting categories are described as follows:

- A Provides clergy training programmes (ie: The Australian and New Zealand Association of Theological Schools [ANZATS] member schools) and/or schools and training institutions which offer broad theological training (ie: Bible Colleges, Missionary Training Institutions) possibly leading to degree or post-graduate qualifications;

- B Do not have students (ie: church administrative libraries, resource centres, parachurch organisations, etc.); and

- C Institutions offering non-theological courses in addition (ie: teacher training).

Data has been entered as submitted by the libraries. Any questions should be directed to the libraries concerned.

Thank you to all libraries who contribute to the ANZTLA Statistics! 
TABLE 12013 LIBRARY INFORMATION

\begin{tabular}{|c|c|c|c|}
\hline $\begin{array}{l}0 \\
m \\
\&\end{array}$ & INSTITUTION & STATE/NZ & $\begin{array}{l}\text { PREDOMINANT DENOMINATION OF } \\
\text { COLLECTION }\end{array}$ \\
\hline A & Adelaide Theological Library & SA & Well-spread over all denominations \\
\hline C & Australian Catholic University & AUST & Catholic \\
\hline A & Australian Lutheran College & SA & Lutheran \\
\hline A & Benedictine Community, New Norcia & WA & Catholic \\
\hline A & Bible College of South Australia & SA & Well-spread over all denominations \\
\hline & Bishopdale Theological College & NZ & Anglican \\
\hline A & Booth College & NSW & Salvation Army \\
\hline A & Booth College of Mission, The Salvation Army & NZ & Salvation Army \\
\hline A & Camden Theological Library, Uniting Church in Australia & NSW & Protestant \\
\hline A & Carey Baptist College & NZ & Protestant, Baptist \\
\hline $\mathrm{B}$ & Carmelite Library, University of Divinity & VIC & Catholic \\
\hline $\mathrm{B}$ & Catholic Education Office of Western Australia & WA & Catholic \\
\hline A & Catholic Theological College & VIC & Catholic \\
\hline A & Chinese Theological College Australia & NSW & Well-spread over all denominations \\
\hline A & Christ College & NSW & Presbyterian \\
\hline C & Christian Heritage College & QLD & Well-spread over all denominations \\
\hline $\mathrm{B}$ & Dominican Fathers & VIC & Catholic \\
\hline A & Emmaus Bible College & NSW & Christian Brethren \\
\hline & Franciscan Order of Friars Minor, St Paschal Library & VIC & Catholic \\
\hline A & Harvest Bible College & VIC & Pentecostal \\
\hline A & Harvest Bible College & QLD & Pentecostal \\
\hline A & John Kinder Theological Library & NZ & Anglican, Methodist \\
\hline A & Knox College, Presbyterian Research Centre, Hewitson & NZ & Well-spread over all denominations \\
\hline C & Lifeway College & NZ & Pentecostal \\
\hline A & Malyon College & QLD & Baptist \\
\hline A & Mary Andrews College & NSW & Well-spread over all denominations \\
\hline A & Melbourne School of Theology & VIC & Well-spread over all denominations \\
\hline A & Morling Theological College & NSW & Baptist \\
\hline A & Nazarene Theological College & QLD & Methodist \\
\hline A & Queensland Theological College & QLD & Presbyterian \\
\hline $\mathrm{A}$ & Ridley Melbourne Mission \& Ministry College & VIC & Anglican \\
\hline A & St. Athanasius Coptic Orthodox Theological College & VIC & Orthodox \\
\hline & St Benedict's Monastery & NSW & Catholic \\
\hline A & St Francis Theological College & QLD & Anglican \\
\hline A & St Mark's National Theological Centre & ACT & Anglican \\
\hline A & Sunshine Coast Theological College & QLD & Protestant, Presbyterian \\
\hline$A$ & Sydney Missionary \& Bible College & NSW & Protestant \\
\hline C & Tabor Adelaide & SA & Well-spread over all denominations \\
\hline C & Tabor Victoria & VIC & Well-spread over all denominations \\
\hline C & Trinity College, The University of Melbourne & VIC & Anglican \\
\hline A & Trinity College & QLD & Protestant \\
\hline A & Trinity Theological College & WA & Well-spread over all denominations \\
\hline A & Vianney College & NSW & Catholic \\
\hline A & Vose Seminary & WA & Baptist \\
\hline C & Wesley Institute & NSW & Well-spread over all denominations \\
\hline A & Youthworks College & NSW & Anglican, Protestant \\
\hline
\end{tabular}




\begin{tabular}{|l|c|l|c|}
\hline Anglican & 7 & Pentecostal & 3 \\
\hline Baptist & 4 & Presbyterian & 3 \\
\hline Catholic & 9 & Protestant & 5 \\
\hline Christian Brethren & 1 & Salvation Army & 2 \\
\hline Lutheran & 1 & Well-spread over all denominations & 11 \\
\hline Methodist & 2 & \multicolumn{2}{|l}{} \\
\cline { 1 - 2 } & \multicolumn{2}{|l|}{} &
\end{tabular}

\section{Predominant Denomination of Collections}

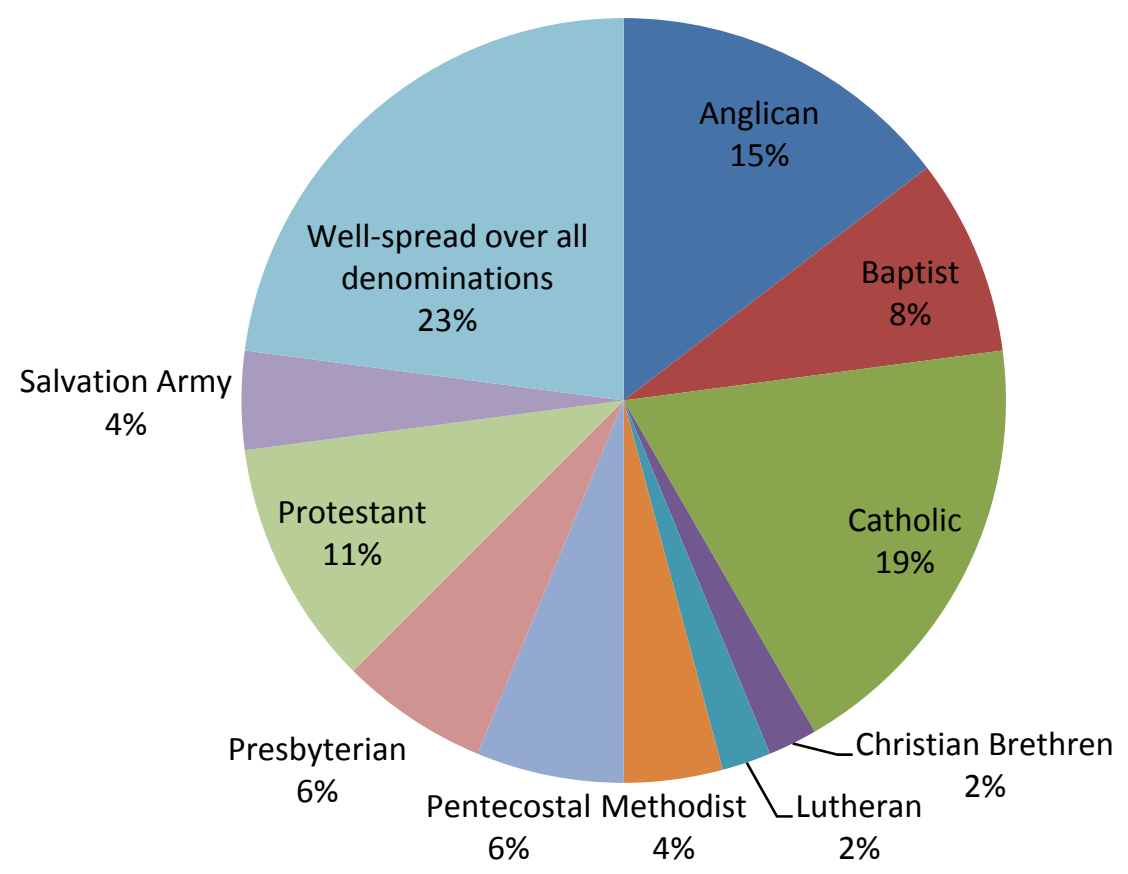


TABLE 22013 LIBRARY STAFF

\begin{tabular}{|c|c|c|c|c|c|c|c|}
\hline \multirow[t]{2}{*}{$\begin{array}{l}0 \\
m \\
4\end{array}$} & \multirow[t]{2}{*}{ INSTITUTION } & \multirow[t]{2}{*}{$\begin{array}{c}\text { STATE/ } \\
\text { NZ }\end{array}$} & $\begin{array}{c}\text { PROF. } \\
\text { POSITIONS }\end{array}$ & $\begin{array}{l}\text { PARA-PROF. } \\
\text { POSITIONS }\end{array}$ & $\begin{array}{c}\text { SUPPORT } \\
\text { STAFF } \\
\text { POSITIONS }\end{array}$ & VOLUNTEERS & TOTAL STAFF \\
\hline & & & [1] & [2] & [3] & [4] & [5] \\
\hline A & Adelaide Theological Library & SA & 1.7 & 0.5 & 0.08 & 0.15 & 2.43 \\
\hline $\mathrm{C}$ & Australian Catholic University & AUST & 42.1 & 31.8 & 19.7 & 0 & 93.60 \\
\hline $\mathrm{A}$ & Australian Lutheran College & $S A$ & 3.35 & 0 & 0.4 & 0 & 3.75 \\
\hline $\mathrm{A}$ & Benedictine Community, New Norcia & WA & 0.8 & 0 & 0 & 0.125 & 0.93 \\
\hline \multirow[t]{2}{*}{$\mathrm{A}$} & Bible College of South Australia & SA & 0.4 & 0 & 0 & 0.5 & 0.90 \\
\hline & Bishopdale Theological College & $\mathrm{NZ}$ & 0.6 & 0.2 & 0 & 0 & 0.80 \\
\hline $\mathrm{A}$ & Booth College & NSW & 2 & 0.12 & 0 & 0 & 2.12 \\
\hline A & Booth College of Mission, The Salvation Army & $\mathrm{NZ}$ & 1 & 0 & 0 & 0 & 1.00 \\
\hline A & Camden Theological Library, Uniting Church in Australia & NSW & 1 & 1.25 & 0.75 & 0 & 3.00 \\
\hline A & Carey Baptist College & NZ & 3 & 0 & 0 & 0 & 3.00 \\
\hline $\mathrm{B}$ & Carmelite Library, University of Divinity & $\mathrm{VIC}$ & 0.8 & 0.2 & 0 & 0.1 & 1.10 \\
\hline $\mathrm{B}$ & Catholic Education Office of Western Australia & WA & 3 & 2 & 2 & 0 & 7.00 \\
\hline A & Catholic Theological College & $\mathrm{VIC}$ & 1.8 & 1 & 0.3 & 0 & 3.10 \\
\hline A & Chinese Theological College Australia & NSW & 0.75 & 0 & 0.25 & 0.25 & 1.25 \\
\hline $\mathrm{A}$ & Christ College & NSW & 0 & 1 & 0 & 0 & 1.00 \\
\hline $\mathrm{C}$ & Christian Heritage College & QLD & 1 & 1 & 1.66 & 0 & 3.66 \\
\hline $\mathrm{B}$ & Dominican Fathers & $\mathrm{VIC}$ & 0.7 & 0 & 0 & 0.05 & 0.75 \\
\hline \multirow[t]{2}{*}{ A } & Emmaus Bible College & NSW & 0.58 & 0.13 & 0 & 0.16 & 0.87 \\
\hline & Franciscan Order of Friars Minor, St Paschal Library & VIC & 1.8 & 0 & 0.6 & 0 & 2.40 \\
\hline A & Harvest Bible College & $\mathrm{VIC}$ & 1 & 0 & 0 & 0 & 1.00 \\
\hline A & Harvest Bible College & QLD & 0 & 0.2 & 0 & 0 & 0.20 \\
\hline A & John Kinder Theological Library & NZ & 5 & 0 & 0 & 2 & 7.00 \\
\hline A & Knox College, Presbyterian Research Centre, Hewitson & NZ & 2 & 0.5 & 0 & 0.5 & 3.00 \\
\hline C & Lifeway College & $\mathrm{NZ}$ & 0.7 & 0 & 0.05 & 0 & 0.75 \\
\hline $\mathrm{A}$ & Malyon College & QLD & 0.86 & 0 & 0 & 0.26 & 1.12 \\
\hline A & Mary Andrews College & NSW & 0 & 0 & 0.33 & 0 & 0.33 \\
\hline A & Melbourne School of Theology & VIC & 1 & 0 & 1.4 & 0.3 & 2.70 \\
\hline $\mathrm{A}$ & Morling Theological College & NSW & 1 & 1.25 & 0 & 1 & 3.25 \\
\hline A & Nazarene Theological College & QLD & 1 & 0 & 1 & 1 & 3.00 \\
\hline A & Queensland Theological College & QLD & 1 & & 0.24 & 0.8 & 2.04 \\
\hline A & Ridley Melbourne Mission \& Ministry College & VIC & 1.8 & 0 & 0.4 & 0 & 2.20 \\
\hline \multirow[t]{2}{*}{ A } & St. Athanasius Coptic Orthodox Theological College & $\mathrm{VIC}$ & 0.5 & 0 & 0 & 0 & 0.50 \\
\hline & St Benedict's Monastery & NSW & 0.2 & 0 & 0 & 0 & 0.20 \\
\hline A & St Francis Theological College & QLD & 1.4 & 0.35 & 0 & 0.19 & 1.94 \\
\hline A & St Mark's National Theological Centre & ACT & 2 & 1 & 1 & 7 & 11.00 \\
\hline A & Sunshine Coast Theological College & QLD & 0 & 0.2 & 0 & 0 & 0.20 \\
\hline $\mathrm{A}$ & Sydney Missionary \& Bible College & NSW & 1.6 & 1 & 0.5 & 1 & 4.10 \\
\hline C & Tabor Adelaide & SA & 2.2 & 0.9 & 0.2 & 0.2 & 3.50 \\
\hline $\mathrm{C}$ & Tabor Victoria & $\mathrm{VIC}$ & 2 & 0.5 & 0 & 0.6 & 3.10 \\
\hline C & Trinity College, The University of Melbourne & $\mathrm{VIC}$ & 1.8 & 1 & 0.33 & 0 & 3.13 \\
\hline A & Trinity College & QLD & 3 & 0 & 0 & 0 & 3.00 \\
\hline A & Trinity Theological College & WA & 1.4 & 0 & 0.3 & 0.2 & 1.90 \\
\hline A & Vianney College & NSW & 0.2 & 1 & 0 & 0 & 1.20 \\
\hline A & Vose Seminary & WA & 1.3 & 0 & 0 & 0.8 & 2.10 \\
\hline C & Wesley Institute & NSW & 1.66 & 0.66 & 0 & 0 & 2.32 \\
\hline A & Youthworks College & NSW & 0 & 0 & 0.3 & 0.2 & 0.50 \\
\hline \multicolumn{3}{|c|}{ TOTAL } & 101 & 47.76 & 31.79 & 17.385 & 197.935 \\
\hline \multicolumn{3}{|c|}{ AVERAGE } & 2.1956522 & 1.0613333 & 0.691087 & 0.37793478 & 4.3029348 \\
\hline
\end{tabular}


TABLE 32013 LIBRARY SERVICES

\begin{tabular}{|c|c|c|c|c|c|c|c|c|c|}
\hline \multirow{3}{*}{$\begin{array}{l}0 \\
0 \\
0\end{array}$} & \multirow{3}{*}{ INSTITUTION } & \multirow{3}{*}{$\begin{array}{c}\text { STATE } / N \\
Z\end{array}$} & \multirow[b]{2}{*}{$\begin{array}{c}\text { TOTAL } \\
\text { LOANS } 2013\end{array}$} & \multicolumn{3}{|c|}{ ILLS SUPPLIED } & \multicolumn{3}{|c|}{ ILLS REQUESTED } \\
\hline & & & & $\begin{array}{c}\text { ORIGINAL } \\
\text { LENT }\end{array}$ & $\begin{array}{l}\text { COPYIFAX } \\
\text { SUPPLIED }\end{array}$ & $\begin{array}{c}\text { TOTAL } \\
\text { SUPPLIED }\end{array}$ & $\begin{array}{l}\text { ITEMS } \\
\text { REQ'D }\end{array}$ & $\begin{array}{c}\text { COPYIFAX } \\
\text { REQ'D }\end{array}$ & $\begin{array}{l}\text { TOTAL } \\
\text { REQ'D }\end{array}$ \\
\hline & & & [6] & [7] & [8] & [9] & [10] & [11] & [12] \\
\hline$A$ & Adelaide Theological Library & $S A$ & 11,756 & 308 & 86 & 394 & 12 & 1 & 13 \\
\hline $\mathrm{C}$ & Australian Catholic University & AUST & 232,382 & 1,403 & 1,688 & 3,091 & 1,094 & 1,456 & 2,550 \\
\hline$A$ & Australian Lutheran College & SA & 12,489 & 40 & 43 & 83 & 8 & 20 & 28 \\
\hline$A$ & Benedictine Community, New Norcia & WA & & 10 & 4 & 14 & 1 & & 1 \\
\hline$A$ & Bible College of South Australia & SA & & & & 0 & 4 & & 4 \\
\hline & Bishopdale Theological College & $\mathrm{NZ}$ & 3,269 & & & 0 & & & 0 \\
\hline $\mathrm{A}$ & Booth College & NSW & 3,210 & & & 0 & & & 0 \\
\hline$A$ & Booth College of Mission, The Salvation Army & NZ & 3,944 & 1 & & 1 & 11 & 1 & 12 \\
\hline$A$ & Camden Theological Library, Uniting Church in Australia & NSW & 6,261 & 18 & 3 & 21 & 9 & & 9 \\
\hline $\mathrm{A}$ & Carey Baptist College & NZ & 11,100 & 51 & 14 & 65 & 40 & 2 & 42 \\
\hline$B$ & Carmelite Library, University of Divinity & VIC & & & & 0 & & & 0 \\
\hline$B$ & Catholic Education Office of Western Australia & WA & 9,016 & & & 0 & & & 0 \\
\hline $\mathrm{A}$ & Catholic Theological College & VIC & 14,004 & 7 & 15 & 22 & 5 & 16 & 21 \\
\hline $\mathrm{A}$ & Chinese Theological College Australia & NSW & & & & 0 & & & 0 \\
\hline $\mathrm{A}$ & Christ College & NSW & 3,430 & & & 0 & & & 0 \\
\hline $\mathrm{C}$ & Christian Heritage College & QLD & 20,494 & 2 & & 2 & 46 & 14 & 60 \\
\hline $\mathrm{B}$ & Dominican Fathers & VIC & 3,000 & & 2 & 2 & & 1 & 1 \\
\hline$A$ & Emmaus Bible College & NSW & 2,597 & 20 & 4 & 24 & 25 & 4 & 29 \\
\hline & Franciscan Order of Friars Minor, St Paschal Library & VIC & 4,060 & & 6 & 6 & 29 & 6 & 35 \\
\hline$A$ & Harvest Bible College & VIC & 5,988 & & & 0 & 3 & 1 & 4 \\
\hline $\mathrm{A}$ & Harvest Bible College & QLD & 621 & & & 0 & & & 0 \\
\hline$A$ & John Kinder Theological Library & NZ & 11,541 & & & 224 & 14 & & 14 \\
\hline $\mathrm{A}$ & Knox College, Presbyterian Research Centre, Hewitson & NZ & 3,087 & & & 13 & & & 0 \\
\hline C & Lifeway College & NZ & 1,156 & & & 0 & & & 0 \\
\hline $\mathrm{A}$ & Malyon College & QLD & 6,201 & 5 & 1 & 6 & 1 & 1 & 2 \\
\hline$A$ & Mary Andrews College & NSW & 1,643 & & & 0 & & & 0 \\
\hline$A$ & Melbourne School of Theology & $\mathrm{VIC}$ & 9,725 & & & 0 & 8 & 13 & 21 \\
\hline A & Morling Theological College & NSW & 22,146 & & & 0 & & & $\underline{0}$ \\
\hline $\mathrm{A}$ & Nazarene Theological College & QLD & & 5 & 2 & 7 & 2 & & 2 \\
\hline $\mathrm{A}$ & Queensland Theological College & QLD & 4,499 & & 1 & 1 & 18 & 10 & 28 \\
\hline$A$ & Ridley Melbourne Mission \& Ministry College & VIC & 13,189 & & 2 & 2 & & 3 & 3 \\
\hline $\mathrm{A}$ & St. Athanasius Coptic Orthodox Theological College & $\mathrm{VIC}$ & & & & 0 & & & 0 \\
\hline & St Benedict's Monastery & NSW & & & & 0 & 3 & & 3 \\
\hline $\mathrm{A}$ & St Francis Theological College & QLD & & 6 & 1 & 7 & & & 0 \\
\hline $\mathrm{A}$ & St Mark's National Theological Centre & ACT & 28,832 & & 581 & 581 & 37 & & 37 \\
\hline $\mathrm{A}$ & Sunshine Coast Theological College & QLD & 169 & & & 0 & & & 0 \\
\hline A & Sydney Missionary \& Bible College & NSW & 13,500 & 2 & 3 & 5 & 3 & 5 & 8 \\
\hline$C$ & Tabor Adelaide & SA & 14,332 & & & 0 & 18 & 3 & 21 \\
\hline C & Tabor Victoria & $\mathrm{VIC}$ & 4,459 & & & 0 & & & 0 \\
\hline $\mathrm{C}$ & Trinity College, The University of Melbourne & VIC & 8,790 & 4 & & 4 & & & 0 \\
\hline $\mathrm{A}$ & Trinity College & QLD & 2,691 & 7 & 2 & 9 & 11 & 3 & 14 \\
\hline$A$ & Trinity Theological College & WA & 1,950 & 2 & 3 & 5 & 8 & 1 & 9 \\
\hline $\mathrm{A}$ & Vianney College & NSW & 1,348 & 2 & & 2 & & 1 & 1 \\
\hline$A$ & Vose Seminary & WA & 6,876 & 6 & 2 & 8 & 3 & 3 & 6 \\
\hline$C$ & Wesley Institute & NSW & & & & 3 & & 3 & 3 \\
\hline $\mathrm{A}$ & Youthworks College & NSW & 389 & & & 0 & & & 0 \\
\hline TOT & TAL & & 504,144 & 1,899 & 2,463 & 4,602 & 1,413 & 1,568 & 2,981 \\
\hline & ERAGE & & 13,626 & 100 & 123 & 100 & 57 & 71 & 65 \\
\hline
\end{tabular}




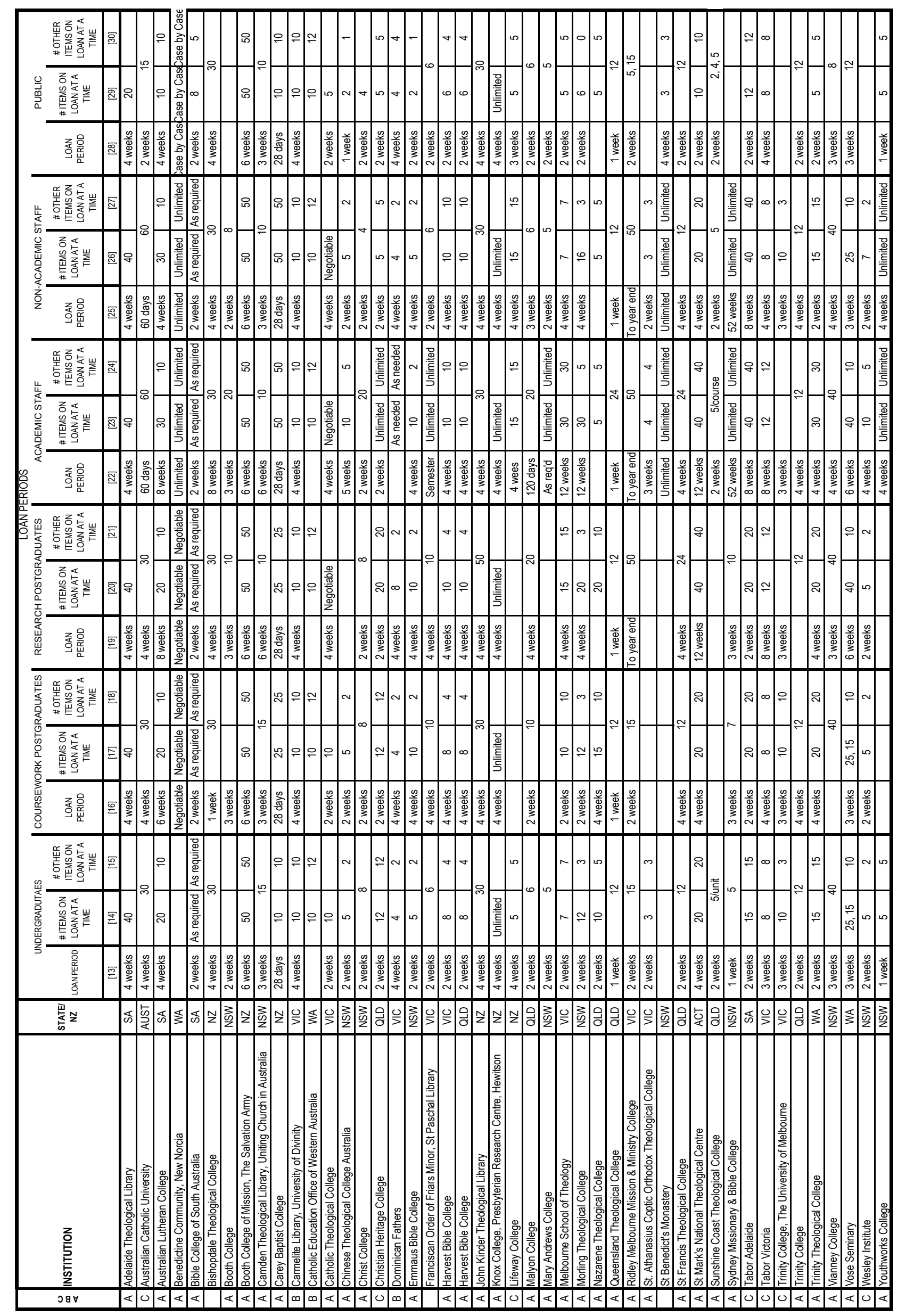


TABLE 52013 COLLECTION RESOURCES

\begin{tabular}{|c|c|c|c|c|c|c|c|c|c|c|c|c|c|}
\hline \multirow{3}{*}{$\begin{array}{l}0 \\
\dot{m} \\
\dot{\alpha}\end{array}$} & \multirow{3}{*}{ INSTITUTION } & \multirow{3}{*}{$\begin{array}{l}\text { STATE/ } \\
\text { NZ }\end{array}$} & \multicolumn{3}{|c|}{ MONOGRAPH VOLUMES } & \multicolumn{3}{|c|}{ NON-BOOK MATERIALS } & \multicolumn{3}{|c|}{ SERIAL VOLUMES } & \multirow[b]{2}{*}{$\begin{array}{l}\text { ELEC. } \\
\text { SERIALS }\end{array}$} & \multirow[b]{2}{*}{\begin{tabular}{|c|} 
ELECT. \\
D'BASES
\end{tabular}} \\
\hline & & & ADDED 2013 & $\begin{array}{c}\text { DONATED } \\
2013\end{array}$ & \begin{tabular}{|c|} 
TOTAL END \\
2013
\end{tabular} & ADDED 2013 & $\begin{array}{c}\text { EBOOKS } \\
\text { ADDED } 2013\end{array}$ & \begin{tabular}{|c|} 
TOTAL END \\
2013
\end{tabular} & $\begin{array}{l}\text { ADDED } \\
2013\end{array}$ & \begin{tabular}{|c|} 
CURRENT \\
SUBS
\end{tabular} & $\begin{array}{c}\text { TOTAL } \\
\text { END } 2013\end{array}$ & & \\
\hline & & & [31] & [32] & [33] & [34] & [35] & [36] & [37] & [38] & [39] & [40] & [41] \\
\hline $\mathrm{A}$ & Adelaide Theological Library & SA & 784 & 416 & 66,055 & & 155 & & 2 & 225 & & 28 & 13 \\
\hline C & Australian Catholic University & AUST & 11,287 & & 416,367 & & 8,136 & 308,564 & 2 & 480 & 759 & 13,419 & 449 \\
\hline A & Australian Lutheran College & SA & 1,172 & 13 & 91,998 & 56 & 18 & 3,959 & 2 & 240 & 720 & & 7 \\
\hline A & Benedictine Community, New Norcia & WA & 240 & 1,473 & 1,713 & & & & & 103 & & & \\
\hline \multirow[t]{2}{*}{$\mathrm{A}$} & Bible College of South Australia & SA & 114 & 931 & 23,698 & 2 & & 274 & & 42 & 387 & 5 & 2 \\
\hline & Bishopdale Theological College & $\mathrm{NZ}$ & & & & & & & & & & & \\
\hline A & Booth College & NSW & 1,818 & & 31,649 & & & & & & 144 & & \\
\hline A & Booth College of Mission, The Salvation Army & NZ & 418 & 151 & 13,982 & 61 & & 2,027 & 2 & 48 & & & 3 \\
\hline A & Camden Theological Library, Uniting Church in Australia & NSW & 3,125 & & 47,049 & & & & & 70 & 559 & & 4 \\
\hline A & Carey Baptist College & NZ & 1,644 & & & & 95 & & 4 & & & 15 & 5 \\
\hline $\mathrm{B}$ & Carmelite Library, University of Divinity & VIC & 4,000 & 3,000 & 40,000 & & & & 2 & 25 & & & \\
\hline$B$ & Catholic Education Office of Western Australia & WA & 508 & 44 & 8,605 & 93 & 1,448 & 2,137 & & 52 & 49 & 1 & 8 \\
\hline A & Catholic Theological College & VIC & 585 & 415 & 131,000 & & & 60 & 2 & 245 & 462 & 12 & 16 \\
\hline A & Chinese Theological College Australia & NSW & 485 & 100 & 4,281 & 5 & & & 4 & 10 & 10 & & \\
\hline A & Christ College & NSW & 1,439 & 16 & 43,357 & 5 & & 1,095 & 192 & 54 & 299 & & 3 \\
\hline C & Christian Heritage College & QLD & 1,413 & & 52,873 & 14 & & 14 & 1 & 68 & 274 & 1 & 7 \\
\hline $\mathrm{B}$ & Dominican Fathers & $\mathrm{VIC}$ & 320 & 60 & & 4 & & & 2 & 48 & 80 & & \\
\hline \multirow[t]{2}{*}{ A } & Emmaus Bible College & NSW & 2,719 & 401 & 3,120 & 1,142 & 5 & 1,147 & & 12 & 62 & & 3 \\
\hline & Franciscan Order of Friars Minor, St Paschal Library & VIC & 734 & 136 & 60,721 & 1 & & 50 & & 303 & 343 & 2 & 5 \\
\hline A & Harvest Bible College & VIC & & & 40,385 & & & & & & & & 5 \\
\hline A & Harvest Bible College & QLD & & & 17,661 & & & & & & & & 5 \\
\hline A & John Kinder Theological Library & $\mathrm{NZ}$ & 2,305 & & & & 220 & & & & & & 4 \\
\hline A & Knox College, Presbyterian Research Centre, Hewitson & NZ & 683 & 1,088 & & & & & & 28 & & & 1 \\
\hline C & Lifeway College & NZ & 51 & 73 & 6,300 & 14 & 2 & 208 & 2 & 11 & 41 & 4 & 1 \\
\hline A & Malyon College & QLD & 1,296 & & 26,154 & 18 & 2 & 1,162 & 9 & 79 & 516 & & 3 \\
\hline A & Mary Andrews College & NSW & 282 & 150 & 6,300 & 11 & & 114 & & 20 & 32 & & \\
\hline A & Melbourne School of Theology & VIC & 1,226 & 297 & 60,000 & 10 & 43 & 43 & 2 & 117 & 205 & & 5 \\
\hline A & Morling Theological College & NSW & & & & & & & & & & & \\
\hline A & Nazarene Theological College & QLD & 50 & 10 & 35,000 & 10 & & 10 & & 44 & 95 & & 1 \\
\hline A & Queensland Theological College & QLD & 1,190 & 16 & & 26 & 1086 & 3,138 & 2 & 37 & 89 & 5 & 5 \\
\hline A & Ridley Melbourne Mission \& Ministry College & VIC & 1,859 & & 54,082 & 14 & 123 & 1,065 & 4 & 161 & 422 & & 5 \\
\hline \multirow[t]{2}{*}{$\mathrm{A}$} & St. Athanasius Coptic Orthodox Theological College & VIC & 81 & 180 & 3,700 & & & & & & & & 3 \\
\hline & St Benedict's Monastery & NSW & 68 & 36 & 20,208 & 54 & & 236 & & 46 & 145 & & \\
\hline A & St Francis Theological College & QLD & & & & & & & & & & 1 & \\
\hline A & St Mark's National Theological Centre & ACT & 807 & 3,528 & 64,672 & 49 & 28 & 508 & 3 & 104 & 404 & & 10 \\
\hline A & Sunshine Coast Theological College & QLD & 146 & 30 & 3,407 & 1 & 2 & 100 & 1 & 18 & 36 & 1 & 1 \\
\hline A & Sydney Missionary \& Bible College & NSW & 981 & 234 & 39,284 & 7 & 140 & 562 & 3 & 139 & 257 & 22 & 6 \\
\hline C & Tabor Adelaide & SA & 1,279 & 564 & 59,000 & 96 & 420 & 516 & 7 & 149 & & 3 & 38 \\
\hline C & Tabor Victoria & VIC & 1,178 & 2,447 & 62,799 & 29 & & 867 & 8 & 70 & 834 & & 4 \\
\hline C & Trinity College, The University of Melbourne & VIC & 503 & 84 & 22,743 & 36 & 122 & 1,279 & 1 & 30 & 29 & 1 & 3 \\
\hline A & Trinity College & QLD & 460 & 322 & 27,663 & 7 & 8 & 15 & 1 & 35 & 137 & 4 & 1 \\
\hline A & Trinity Theological College & WA & 1,145 & 50 & 25,007 & 13 & 1 & 14 & & 70 & 141 & 5 & 10 \\
\hline A & Vianney College & NSW & 336 & & 336 & & & & 1 & 59 & 107 & & 1 \\
\hline A & Vose Seminary & WA & 475 & 1,559 & 42,467 & 37 & 176 & 48,300 & & 80 & & & 4 \\
\hline $\mathrm{C}$ & Wesley Institute & NSW & & 25 & 31,485 & 1,340 & & & & & 209 & & 13 \\
\hline A & Youthworks College & NSW & 364 & & 4,915 & 9 & & 166 & & 15 & 56 & & \\
\hline \multicolumn{2}{|c|}{ TOTAL } & & 49,570 & 17,849 & $1,690,036$ & 3,164 & 12,230 & 377,630 & 259 & 3,337 & 7,903 & 13,529 & 654 \\
\hline \multicolumn{2}{|c|}{ AVERAGE } & & 1,239 & 576 & 44,475 & 109 & 612 & 13,487 & 11 & 93 & 255 & 796 & 19 \\
\hline
\end{tabular}


TABLE 62013 LIBRARY EXPENDITURE

\begin{tabular}{|c|c|c|c|c|c|c|c|c|c|}
\hline \multirow[t]{2}{*}{$\begin{array}{l}0 \\
0 \\
4\end{array}$} & \multirow[t]{2}{*}{ INSTITUTION } & \multirow[t]{2}{*}{$\begin{array}{c}\text { STATEI } \\
\text { NZ }\end{array}$} & MONOGRAPHS & $\begin{array}{l}\text { NON-BOOK } \\
\text { MATERIALS }\end{array}$ & EBOOKS & SERIAL SUBS. & $\begin{array}{l}\text { ELECTRONIC SERIAL } \\
\text { SUBS. }\end{array}$ & $\begin{array}{c}\text { ELECTRONIC } \\
\text { DATABASE SUBS. }\end{array}$ & TOTAL SPENT \\
\hline & & & [42] & [43] & [44] & [45] & [46] & [47] & [48] \\
\hline $\bar{A}$ & Adelaide Theological Library & $\mathrm{SA}$ & $\$ 36,447$ & & & $\$ 28,553$ & & $\$ 11,836$ & $\$ 76,836$ \\
\hline C & Australian Catholic University & AUST & $\$ 822,165$ & & $\$ 945,652$ & $\$ 55,035$ & & $\$ 2,461,029$ & $\$ 4,294,881$ \\
\hline $\mathrm{A}$ & Australian Lutheran College & SA & $\$ 29,125$ & 1250 & 850 & $\$ 17,150$ & & $\$ 8,350$ & $\$ 55,875$ \\
\hline$A$ & Benedictine Community, New Norcia & WA & & & & & & & $\$ 10,000$ \\
\hline $\mathrm{A}$ & Bible College of South Australia & SA & $\$ 4,019$ & & & $\$ 3,093$ & & 1403 & \\
\hline & Bishopdale Theological College & NZ & $\$ 10,000$ & $\$ 2,000$ & & $\$ 1,800$ & & & $\$ 13,800$ \\
\hline $\mathrm{A}$ & Booth College & NSW & $\$ 35,000$ & & & $\$ 13,000$ & & & $\$ 48,000$ \\
\hline$A$ & Booth College of Mission, The Salvation Army & $\mathrm{NZ}$ & $\$ 11,234$ & & & $\$ 3,863$ & & $\$ 1,390$ & $\$ 17,572$ \\
\hline A & Camden Theological Library, Uniting Church in Australia & NSW & $\$ 84,000$ & & & $\$ 12,500$ & & $\$ 12,500$ & $\$ 109,000$ \\
\hline A & Carey Baptist College & $\mathrm{NZ}$ & $\$ 35,439$ & & $\$ 4,619$ & $\$ 8,558$ & & $\$ 14,399$ & $\$ 63,016$ \\
\hline $\mathrm{B}$ & Carmelite Library, University of Divinity & VIC & & & & & & & \\
\hline $\mathrm{B}$ & Catholic Education Office of Western Australia & WA & $\$ 10,000$ & $\$ 7,500$ & $\$ 10,000$ & $\$ 13,000$ & $\$ 400$ & $\$ 30,000$ & $\$ 71,000$ \\
\hline A & Catholic Theological College & VIC & $\$ 41,700$ & & & $\$ 36,250$ & & $\$ 10,500$ & $\$ 88,450$ \\
\hline A & Chinese Theological College Australia & NSW & $\$ 8,000$ & & & $\$ 100$ & & & $\$ 8,100$ \\
\hline A & Christ College & NSW & $\$ 20,000$ & & & $\$ 6,810$ & & $\$ 6,848$ & $\$ 33,659$ \\
\hline $\mathrm{C}$ & Christian Heritage College & QLD & $\$ 48,304$ & $\$ 496$ & & $\$ 1,000$ & $\$ 300$ & $\$ 17,000$ & $\$ 67,100$ \\
\hline$B$ & Dominican Fathers & VIC & & & & & & & \\
\hline A & Emmaus Bible College & NSW & & & & & & & \\
\hline & Franciscan Order of Friars Minor, St Paschal Library & $\mathrm{VIC}$ & $\$ 31,000$ & $\$ 100$ & & $\$ 33,000$ & & $\$ 4,500$ & $\$ 70,000$ \\
\hline A & Harvest Bible College & $\mathrm{VIC}$ & & & & & & & $\$ 27,132$ \\
\hline A & Harvest Bible College & QLD & & & & & & & $\$ 2,440$ \\
\hline A & John Kinder Theological Library & NZ & & & & & & & \\
\hline A & Knox College, Presbyterian Research Centre, Hewitson & $\mathrm{NZ}$ & $\$ 4,141$ & & & $\$ 2,700$ & & $\$ 744$ & $\$ 7,555$ \\
\hline $\mathrm{C}$ & Lifeway College & NZ & $\$ 1,812$ & $\$ 1,233$ & & $\$ 1,758$ & & $\$ 64$ & $\$ 4,880$ \\
\hline A & Malyon College & QLD & $\$ 39,968$ & $\$ 45$ & & $\$ 6,228$ & & $\$ 8,082$ & $\$ 35,117$ \\
\hline A & Mary Andrews College & NSW & & & & & & & $\$ 10,563$ \\
\hline A & Melbourne School of Theology & VIC & $\$ 16,084$ & $\$ 101$ & $\$ 769$ & $\$ 9,956$ & & $\$ 6,164$ & $\$ 33,074$ \\
\hline A & Morling Theological College & NSW & & & & & & & \\
\hline A & Nazarene Theological College & QLD & $\$ 4,275$ & $\$ 250$ & & $\$ 8,500$ & & $\$ 1,500$ & $\$ 15,000$ \\
\hline A & Queensland Theological College & QLD & $\$ 37,293$ & $\$ 7,645$ & & $\$ 1,217$ & $\$ 22$ & $\$ 8,710$ & $\$ 54,889$ \\
\hline A & Ridley Melbourne Mission \& Ministry College & VIC & $\$ 59,074$ & & $\$ 4,287$ & $\$ 11,597$ & & $\$ 8,223$ & $\$ 83,182$ \\
\hline A & St. Athanasius Coptic Orthodox Theological College & VIC & $\$ 2,608$ & & & & & & $\$ 2,608$ \\
\hline & St Benedict's Monastery & NSW & $\$ 2,360$ & $\$ 300$ & & $\$ 2,059$ & & & $\$ 4,719$ \\
\hline A & St Francis Theological College & QLD & & & & & & & \\
\hline A & St Mark's National Theological Centre & ACT & $\$ 37,976$ & $\$ 1,200$ & $\$ 1,313$ & $\$ 13,451$ & & $\$ 8,878$ & $\$ 62,818$ \\
\hline$A$ & Sunshine Coast Theological College & QLD & $\$ 1,817$ & & & $\$ 390$ & & $\$ 1,134$ & $\$ 3,341$ \\
\hline A & Sydney Missionary \& Bible College & NSW & $\$ 40,947$ & $\$ 50$ & $\$ 3,594$ & $\$ 8,235$ & $\$ 6,000$ & $\$ 8,723$ & $\$ 67,549$ \\
\hline $\mathrm{C}$ & Tabor Adelaide & SA & & & & & & & \\
\hline $\mathrm{C}$ & Tabor Victoria & VIC & $\$ 35,869$ & $\$ 153$ & $\$ 177$ & $\$ 15,392$ & $\$ 7,681$ & $\$ 7,726$ & $\$ 59,120$ \\
\hline $\mathrm{C}$ & Trinity College, The University of Melbourne & VIC & $\$ 18,030$ & $\$ 2,096$ & $\$ 5,645$ & $\$ 5,255$ & $\$ 60$ & $\$ 4,296$ & $\$ 35,374$ \\
\hline A & Trinity College & QLD & $\$ 33,728$ & $\$ 484$ & $\$ 495$ & $\$ 2,634$ & $\$ 308$ & $\$ 100$ & $\$ 37,750$ \\
\hline A & Trinity Theological College & WA & $\$ 35,270$ & $\$ 139$ & $\$ 51$ & $\$ 5,638$ & & $\$ 11,847$ & $\$ 52,947$ \\
\hline A & Vianney College & NSW & $\$ 2,296$ & & & $\$ 3,992$ & & $\$ 150$ & $\$ 6,288$ \\
\hline A & Vose Seminary & WA & $\$ 14,667$ & $\$ 740$ & $\$ 8,357$ & $\$ 8,240$ & & $\$ 500$ & $\$ 41,500$ \\
\hline $\mathrm{C}$ & Wesley Institute & NSW & $\$ 12,200$ & & & $\$ 3,599$ & & $\$ 18,000$ & $\$ 33,799$ \\
\hline $\mathrm{A}$ & Youthworks College & NSW & & & & & & & \\
\hline TOT & TAL & & $\$ 1,626,848$ & $\$ 25,782$ & & $\$ 344,553$ & $\$ 14,771$ & $\$ 2,674,596$ & $\$ 5,708,934$ \\
\hline & ERAGE & & $\$ 47,848$ & $\$ 1,432$ & & $\$ 10,441$ & $\$ 2,110$ & $\$ 92,227$ & $\$ 154,296$ \\
\hline
\end{tabular}

Note: NZ Libraries use NZ\$, all other figures are AUD\$ 
TABLE 72013 INSTITUTIONAL POPULATION

\begin{tabular}{|c|c|c|c|c|c|c|c|c|c|c|c|}
\hline \multirow[t]{2}{*}{$\begin{array}{l}0 \\
m \\
\&\end{array}$} & \multirow[t]{2}{*}{ INSTITUTION } & \multirow[t]{2}{*}{\begin{tabular}{|c} 
STATE/ \\
NZ
\end{tabular}} & $\begin{array}{l}\text { ACADEM } \\
\text { FULL TIME }\end{array}$ & $\begin{array}{l}\text { IIC STAFF } \\
\text { PART TIME }\end{array}$ & $\begin{array}{l}\text { NON-ACADE } \\
\text { FULL TIME }\end{array}$ & $\begin{array}{l}\text { EMIC STAFF } \\
\text { PART TIME }\end{array}$ & FULL TIME & $\begin{array}{l}\text { STUDENT PC } \\
\text { PART TIME }\end{array}$ & $\begin{array}{l}\text { OPULATION } \\
\begin{array}{l}\text { TOTAL \# OF } \\
\text { STUDENTS }\end{array}\end{array}$ & EFT & $\begin{array}{l}\text { REGISTERED } \\
\text { BORROWERS }\end{array}$ \\
\hline & & & [49] & {$[50]$} & [51] & [52] & [53] & [54] & [55] & {$[56]$} & {$[57]$} \\
\hline A & Adelaide Theological Library & SA & 25 & 65 & 10 & 9 & & & 288 & 87 & 189 \\
\hline $\mathrm{C}$ & Australian Catholic University & AUST & & & & & & & & & \\
\hline $\mathrm{A}$ & Australian Lutheran College & $\mathrm{SA}$ & 13 & & 17 & 14 & 44 & 169 & 213 & 77 & 33 \\
\hline A & Benedictine Community, New Norcia & WA & 11 & 1 & & & & & & & \\
\hline \multirow[t]{2}{*}{$\mathrm{A}$} & Bible College of South Australia & $\mathrm{SA}$ & 5 & 3 & 2 & 3 & & & & & \\
\hline & Bishopdale Theological College & NZ & 3 & 9 & & 2 & 8 & 31 & 39 & & 205 \\
\hline A & Booth College & NSW & 8 & 15 & 6 & 8 & 40 & 95 & 135 & 50 & \\
\hline A & Booth College of Mission, The Salvation Army & $\mathrm{NZ}$ & 9 & 2 & 9 & 4 & 20 & 3 & 23 & 19 & 374 \\
\hline A & Camden Theological Library, Uniting Church in Australia & NSW & & & & & & & & & \\
\hline A & Carey Baptist College & $\mathrm{NZ}$ & 8 & 10 & 13 & 5 & 170 & 133 & 303 & 139 & 20 \\
\hline$B$ & Carmelite Library, University of Divinity & $\mathrm{VIC}$ & & & & & & & & & 480 \\
\hline$B$ & Catholic Education Office of Western Australia & WA & & & & & & & & & 1,414 \\
\hline $\mathrm{A}$ & Catholic Theological College & VIC & 6 & 36 & 4 & 1 & 87 & 263 & 350 & 162 & 467 \\
\hline A & Chinese Theological College Australia & NSW & 3 & 1 & 1 & 3 & 5 & 35 & 40 & 30 & 150 \\
\hline $\mathrm{A}$ & Christ College & NSW & 5 & 16 & 1 & 3 & 62 & 157 & 219 & 130 & 70 \\
\hline $\mathrm{C}$ & Christian Heritage College & QLD & & & & & & & & & \\
\hline$B$ & Dominican Fathers & $\mathrm{VIC}$ & & 3 & & 1 & 10 & & 10 & 10 & 350 \\
\hline \multirow[t]{2}{*}{ A } & Emmaus Bible College & NSW & 5 & 6 & 2 & 2 & & & 84 & 37 & 542 \\
\hline & Franciscan Order of Friars Minor, St Paschal Library & $\mathrm{VIC}$ & 2 & 27 & 1 & 5 & 39 & 216 & 255 & 76 & 144 \\
\hline A & Harvest Bible College & $\mathrm{VIC}$ & & & & & & & \multirow{2}{*}{783} & 327 & 8 \\
\hline A & Harvest Bible College & QLD & & & & & & & & 81 & 5 \\
\hline $\mathrm{A}$ & John Kinder Theological Library & NZ & & & & & 35 & 38 & & & \\
\hline A & Knox College, Presbyterian Research Centre, Hewitson & NZ & 3 & 1 & 1 & & & & & & 1,023 \\
\hline $\mathrm{C}$ & Lifeway College & NZ & & & & & & & & & \\
\hline A & Malyon College & QLD & 6 & 4 & 2 & 1 & 74 & 150 & 224 & 130 & 38 \\
\hline $\mathrm{A}$ & Mary Andrews College & NSW & 1 & 7 & 1 & 3 & 2 & 154 & 156 & 37 & 15 \\
\hline A & Melbourne School of Theology & $\mathrm{VIC}$ & 13 & 2 & 9 & 9 & 72 & 221 & 293 & 149 & 35 \\
\hline A & Morling Theological College & NSW & & & & & & & & 180 & \\
\hline A & Nazarene Theological College & QLD & 3 & 3 & & 1 & 7 & 15 & 22 & 9 & 30 \\
\hline $\mathrm{A}$ & Queensland Theological College & QLD & 5 & 7 & 1 & 4 & 52 & 71 & 123 & 75 & 453 \\
\hline $\mathrm{A}$ & Ridley Melbourne Mission \& Ministry College & VIC & 8 & 11 & 4 & 9 & 83 & 218 & 301 & 134 & 120 \\
\hline \multirow[t]{2}{*}{ A } & St. Athanasius Coptic Orthodox Theological College & VIC & 1 & 3 & 1 & & & 69 & 69 & & \\
\hline & St Benedict's Monastery & NSW & 6 & & & & & & & & 23 \\
\hline $\mathrm{A}$ & St Francis Theological College & QLD & 4 & 12 & & 1 & 8 & 112 & 120 & 60 & \\
\hline A & St Mark's National Theological Centre & $\mathrm{ACT}$ & 7 & 29 & 2 & 6 & & & & & 131 \\
\hline $\mathrm{A}$ & Sunshine Coast Theological College & QLD & 1 & 1 & 1 & 1 & 1 & 6 & 7 & 3 & 9 \\
\hline $\mathrm{A}$ & Sydney Missionary \& Bible College & NSW & 20 & 3 & 19 & 3 & 159 & 325 & 484 & 231 & \\
\hline $\mathrm{C}$ & Tabor Adelaide & SA & 23 & & 18 & & & & 1,090 & 409 & \\
\hline $\mathrm{C}$ & Tabor Victoria & $\mathrm{VIC}$ & & & & & & & & & \\
\hline $\mathrm{C}$ & Trinity College, The University of Melbourne & $\mathrm{VIC}$ & & & & & & & & & \\
\hline $\mathrm{A}$ & Trinity College & QLD & 5 & 2 & 2 & 1 & 7 & 11 & 12 & 2 & 336 \\
\hline A & Trinity Theological College & WA & 5 & 1 & 2 & 10 & 23 & 57 & 80 & 35 & 154 \\
\hline $\mathrm{A}$ & Vianney College & NSW & 3 & 10 & 1 & 4 & 20 & & 20 & & 141 \\
\hline $\mathrm{A}$ & Vose Seminary & WA & & & & & & & & & \\
\hline $\mathrm{C}$ & Wesley Institute & NSW & 11 & 2 & 14 & 8 & 288 & 271 & 559 & 324 & \\
\hline$A$ & Youthworks College & NSW & & & & & & & 137 & & 145 \\
\hline \multicolumn{2}{|c|}{ TOTAL } & & 228 & 291.8 & 144 & 120.5 & 1316 & 2820 & 6439 & 3003 & 7104 \\
\hline \multicolumn{2}{|c|}{ AVERAGE } & & 7.3548387 & 10.062069 & 5.5384615 & 4.462963 & 54.833333 & 122.6087 & 222.03448 & 111.2222 & 244.9655172 \\
\hline
\end{tabular}


TABLE 82013 BIBLIOGRAPHIC INSTRUCTION

\begin{tabular}{|c|c|c|c|c|}
\hline \multirow{3}{*}{$\begin{array}{l}0 \\
0 \\
\&\end{array}$} & \multirow{3}{*}{ INSTITUTION } & \multirow{3}{*}{$\begin{array}{c}\text { STATE/N } \\
\mathrm{Z}\end{array}$} & \multicolumn{2}{|c|}{ BIBLIOGRAPHIC INSTRUCTION } \\
\hline & & & $\begin{array}{c}\text { NO. OF } \\
\text { ATTENDEES }\end{array}$ & $\begin{array}{c}\text { NO. OF } \\
\text { SESSIONS }\end{array}$ \\
\hline & & & [58] & [59] \\
\hline A & Adelaide Theological Library & SA & 16 & 4 \\
\hline $\mathrm{C}$ & Australian Catholic University & AUST & 18844 & 656 \\
\hline $\bar{A}$ & Australian Lutheran College & SA & 35 & 5 \\
\hline $\bar{A}$ & Benedictine Community, New Norcia & WA & & \\
\hline$A$ & Bible College of South Australia & SA & & \\
\hline & Bishopdale Theological College & NZ & 20 & 2 \\
\hline A & Booth College & NSW & 40 & \\
\hline$A$ & Booth College of Mission, The Salvation Army & NZ & 9 & 1 \\
\hline$A$ & Camden Theological Library, Uniting Church in Australia & NSW & & \\
\hline A & Carey Baptist College & NZ & 130 & 16 \\
\hline$B$ & Carmelite Library, University of Divinity & VIC & 40 & 6 \\
\hline$B$ & Catholic Education Office of Western Australia & WA & & \\
\hline $\bar{A}$ & Catholic Theological College & VIC & & \\
\hline A & Chinese Theological College Australia & NSW & 15 & 2 \\
\hline A & Christ College & NSW & 40 & 1 \\
\hline C & Christian Heritage College & QLD & 150 & 5 \\
\hline$B$ & Dominican Fathers & VIC & 4 & 1 \\
\hline$A$ & Emmaus Bible College & NSW & 30 & 6 \\
\hline & Franciscan Order of Friars Minor, St Paschal Library & VIC & 50 & 4 \\
\hline A & Harvest Bible College & VIC & & \\
\hline$A$ & Harvest Bible College & QLD & & \\
\hline $\bar{A}$ & John Kinder Theological Library & NZ & 20 & 1 \\
\hline A & Knox College, Presbyterian Research Centre, Hewitson & NZ & & \\
\hline $\mathrm{C}$ & Lifeway College & NZ & 150 & 12 \\
\hline A & Malyon College & QLD & 45 & 2 \\
\hline A & Mary Andrews College & NSW & & \\
\hline A & Melbourne School of Theology & VIC & & \\
\hline A & Morling Theological College & NSW & & \\
\hline$A$ & Nazarene Theological College & QLD & & \\
\hline $\bar{A}$ & Queensland Theological College & QLD & 48 & 2 \\
\hline$A$ & Ridley Melbourne Mission \& Ministry College & VIC & 74 & 21 \\
\hline$A$ & St. Athanasius Coptic Orthodox Theological College & VIC & 50 & 3 \\
\hline & St Benedict's Monastery & NSW & & \\
\hline A & St Francis Theological College & QLD & & \\
\hline$A$ & St Mark's National Theological Centre & ACT & 250 & 20 \\
\hline A & Sunshine Coast Theological College & QLD & 2 & 2 \\
\hline A & Sydney Missionary \& Bible College & NSW & 508 & 266 \\
\hline $\mathrm{C}$ & Tabor Adelaide & SA & & \\
\hline $\mathrm{C}$ & Tabor Victoria & VIC & 65 & 24 \\
\hline $\mathrm{C}$ & Trinity College, The University of Melbourne & VIC & 4 & 2 \\
\hline$A$ & Trinity College & QLD & & \\
\hline$A$ & Trinity Theological College & WA & 30 & 2 \\
\hline$A$ & Vianney College & NSW & & \\
\hline$A$ & Vose Seminary & WA & & \\
\hline $\mathrm{C}$ & Wesley Institute & NSW & 250 & 12 \\
\hline$A$ & Youthworks College & NSW & & \\
\hline T01 & $\mathrm{AL}$ & & 20919 & 1078 \\
\hline$\overline{A V E}$ & RAGE & & 774.7777778 & 41.46153846 \\
\hline
\end{tabular}




\section{TABLE 92013 LIBRARY FACILITIES}

\begin{tabular}{|c|c|c|c|c|c|c|c|}
\hline \multirow[t]{2}{*}{$\begin{array}{l}0 \\
\text { m } \\
\ll\end{array}$} & \multirow[t]{2}{*}{ INSTITUTION } & \multirow[t]{2}{*}{$\begin{array}{c}\text { STATE/N } \\
Z\end{array}$} & \multirow[t]{2}{*}{ N $\begin{array}{c}\text { TOTAL SEATING } \\
\text { CAPACITY }\end{array}$} & \multirow{2}{*}{\begin{tabular}{|c|}
$\begin{array}{c}\text { COMPUTERS } \\
\text { FOR } \\
\text { STUDENTS }\end{array}$ \\
{$[61]$}
\end{tabular}} & \multirow{2}{*}{$\begin{array}{c}\begin{array}{c}\text { COMPUTERS } \\
\text { WITH } \\
\text { INTERNET }\end{array} \\
{[62]} \\
\end{array}$} & \multirow{2}{*}{\begin{tabular}{|c}
$\begin{array}{c}\text { WIRELESS } \\
\text { AVAILABLE? }\end{array}$ \\
{$[63]$}
\end{tabular}} & \multirow{2}{*}{$\begin{array}{c}\text { COMPUTER } \\
\text { DOCKING } \\
\text { STATIONS } \\
{[64]}\end{array}$} \\
\hline & & & & & & & \\
\hline A & Adelaide Theological Library & SA & 40 & 8 & 12 & No & 0 \\
\hline C & Australian Catholic University & AUST & 2024 & 770 & 887 & Yes & 62 \\
\hline$A$ & Australian Lutheran College & SA & 65 & 6 & 13 & Yes & 1 \\
\hline A & Benedictine Community, New Norcia & WA & 60 & & 3 & & \\
\hline \multirow[t]{2}{*}{$\mathrm{A}$} & Bible College of South Australia & SA & & & & Yes & 0 \\
\hline & Bishopdale Theological College & $\mathrm{NZ}$ & 5 & 2 & 3 & Yes & \\
\hline A & Booth College & NSW & 49 & 11 & 13 & Yes & \\
\hline A & Booth College of Mission, The Salvation Army & $\mathrm{NZ}$ & 37 & 5 & 5 & Yes & 22 \\
\hline A & Camden Theological Library, Uniting Church in Australia & NSW & 30 & 11 & 16 & Yes & 20 \\
\hline A & Carey Baptist College & NZ & 113 & 16 & 20 & Yes & 40 \\
\hline $\mathrm{B}$ & Carmelite Library, University of Divinity & $\mathrm{VIC}$ & 30 & 2 & 0 & No & 0 \\
\hline $\mathrm{B}$ & Catholic Education Office of Western Australia & WA & 26 & 2 & 5 & Yes & 0 \\
\hline A & Catholic Theological College & VIC & 70 & 11 & 15 & Yes & \\
\hline A & Chinese Theological College Australia & NSW & & & & Yes & \\
\hline A & Christ College & NSW & 27 & 6 & 9 & Yes & 9 \\
\hline $\mathrm{C}$ & Christian Heritage College & QLD & 115 & 24 & 12 & Yes & 0 \\
\hline B & Dominican Fathers & VIC & 12 & 5 & 5 & No & 3 \\
\hline \multirow[t]{2}{*}{ A } & Emmaus Bible College & NSW & 14 & 3 & 7 & Yes & 12 \\
\hline & Franciscan Order of Friars Minor, St Paschal Library & VIC & 40 & 3 & 8 & Yes & 6 \\
\hline A & Harvest Bible College & $\mathrm{VIC}$ & 22 & 8 & 9 & Yes & 0 \\
\hline A & Harvest Bible College & QLD & 5 & 4 & 5 & No & 0 \\
\hline A & John Kinder Theological Library & $\mathrm{NZ}$ & 51 & 5 & 17 & Yes & 0 \\
\hline A & Knox College, Presbyterian Research Centre, Hewitson & $\mathrm{NZ}$ & 58 & 4 & 4 & Yes & 2 \\
\hline $\mathrm{C}$ & Lifeway College & $\mathrm{NZ}$ & 24 & 7 & 8 & No & 12 \\
\hline A & Malyon College & QLD & 80 & 7 & 9 & Yes & 20 \\
\hline A & Mary Andrews College & NSW & 14 & 2 & 2 & No & 0 \\
\hline A & Melbourne School of Theology & VIC & 69 & 6 & 10 & Yes & \\
\hline A & Morling Theological College & NSW & & 13 & 9 & Yes & \\
\hline$A$ & Nazarene Theological College & QLD & 25 & 5 & 8 & Yes & 6 \\
\hline A & Queensland Theological College & QLD & 39 & 4 & 12 & Yes & \\
\hline A & Ridley Melbourne Mission \& Ministry College & $\mathrm{VIC}$ & 80 & 10 & 14 & Yes & 26 \\
\hline \multirow[t]{2}{*}{$\mathrm{A}$} & St. Athanasius Coptic Orthodox Theological College & $\mathrm{VIC}$ & 8 & 3 & 4 & No & 0 \\
\hline & St Benedict's Monastery & NSW & 20 & 0 & 1 & No & 1 \\
\hline A & St Francis Theological College & QLD & 23 & 6 & 9 & Yes & 2 \\
\hline A & St Mark's National Theological Centre & ACT & 15 & 5 & 11 & Yes & 0 \\
\hline A & Sunshine Coast Theological College & QLD & 4 & 1 & 2 & Yes & \\
\hline $\mathrm{A}$ & Sydney Missionary \& Bible College & NSW & 90 & 5 & 9 & Yes & 55 \\
\hline C & Tabor Adelaide & SA & 39 & 7 & 14 & Yes & 23 \\
\hline $\mathrm{C}$ & Tabor Victoria & VIC & 44 & 9 & 13 & Yes & 0 \\
\hline C & Trinity College, The University of Melbourne & VIC & 120 & 13 & 17 & Yes & 28 \\
\hline A & Trinity College & QLD & 32 & 3 & 3 & Yes & 0 \\
\hline A & Trinity Theological College & WA & 52 & 6 & 8 & Yes & 16 \\
\hline A & Vianney College & NSW & 17 & 5 & 7 & Yes & 0 \\
\hline A & Vose Seminary & WA & & & & Yes & \\
\hline C & Wesley Institute & NSW & 46 & 19 & 19 & Yes & 24 \\
\hline A & Youthworks College & NSW & 2 & 2 & 2 & Yes & 0 \\
\hline \multicolumn{2}{|c|}{ TOTAL } & & 3736 & 1044 & 1259 & & 390 \\
\hline \multicolumn{3}{|c|}{ AVERAGE } & 88.95238095 & 24.85714286 & 29.27906977 & & 10.83333333 \\
\hline
\end{tabular}


TABLE 102013 STAFF SALARIES

\begin{tabular}{|c|c|c|c|}
\hline INSTITUTION & PROF. & CHURCH & OTHER \\
\hline A & 20 & 6 & 4 \\
\hline B & 2 & 1 & 2 \\
\hline C & 3 & 1 & 3 \\
\hline TOTAL & 25 & 8 & 9 \\
\hline
\end{tabular}

Staff Salaries

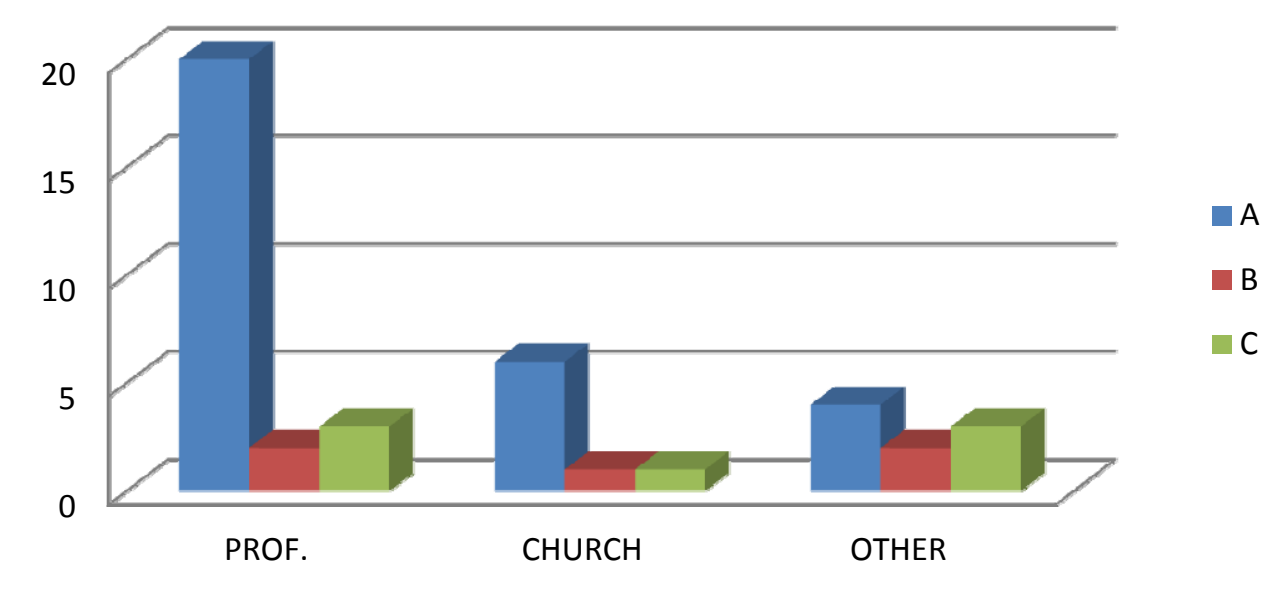




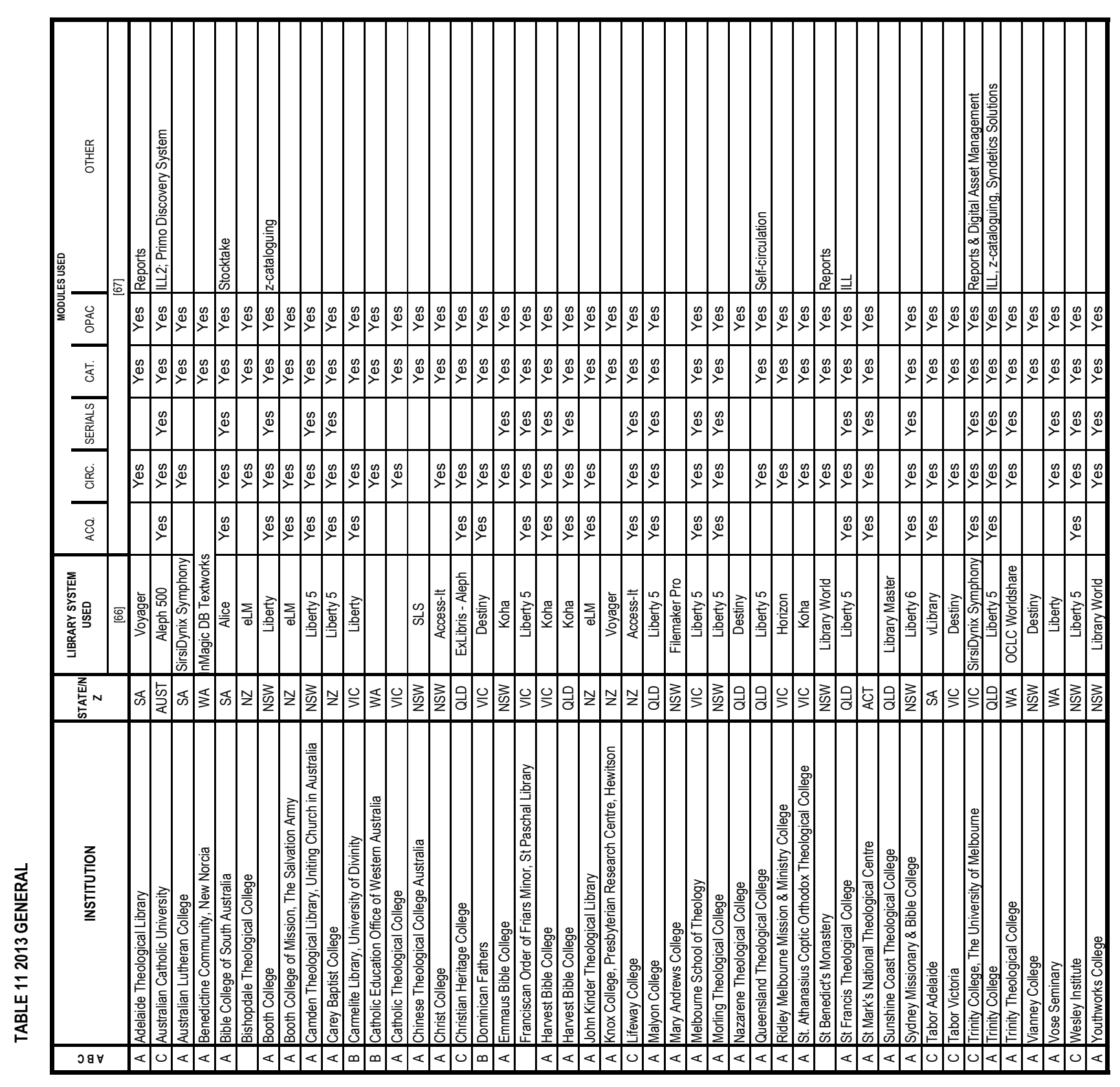




\section{Library System Used}

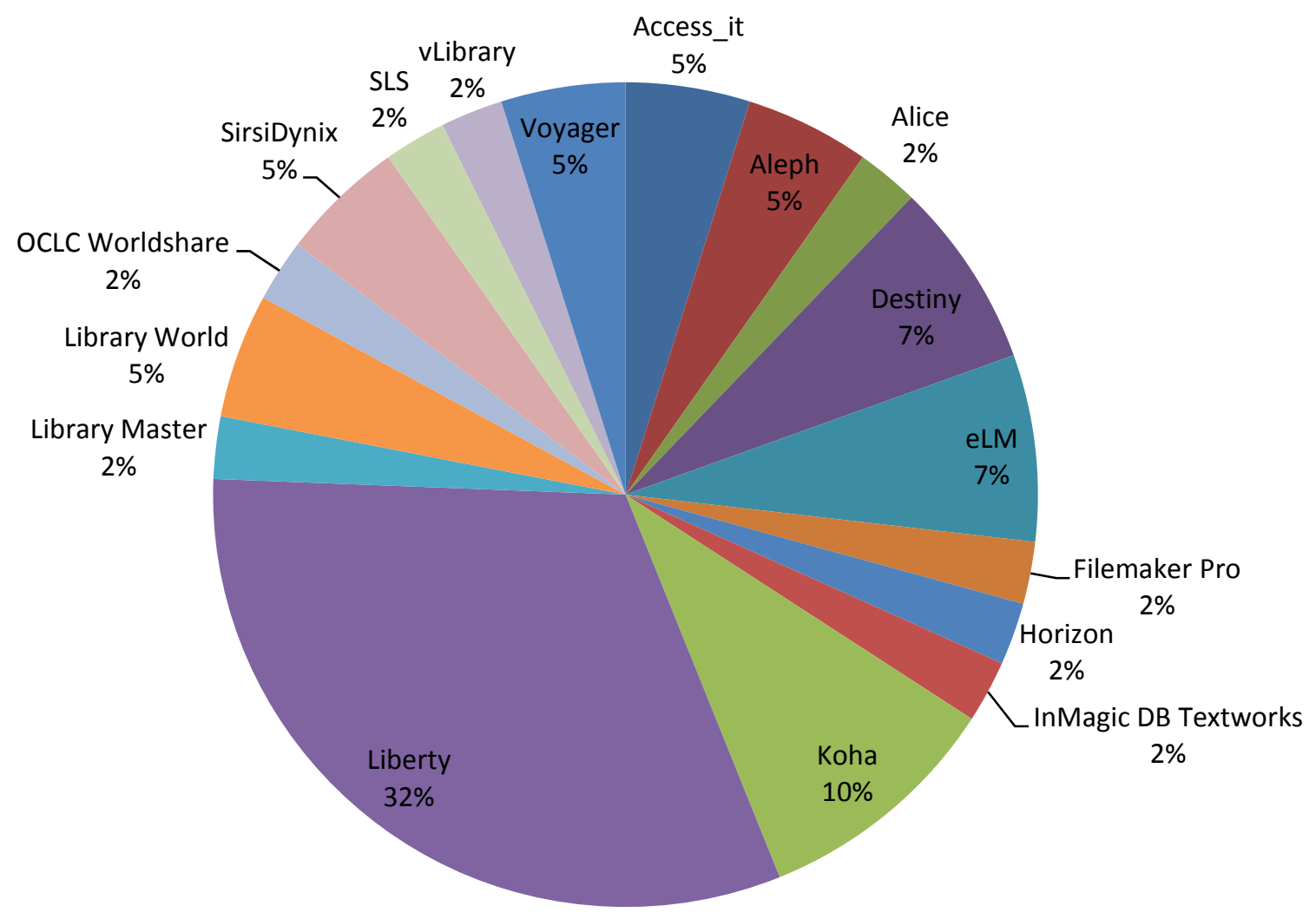

